\title{
PROPERTIES OF HORIZONTALLY ORIENTED ICE CRYSTALS OBSERVED BY POLARIZATION LIDAR OVER SUMMIT, GREENLAND RYAN R. NEELY III ${ }^{1,2, *}$, ROBERT A. STILLWELL ${ }^{3}$, SEBASTIAN COLE ${ }^{1}$, JEFFREY P. THAYER ${ }^{3}$ MATTHEW SHUPE ${ }^{2,4}$, MARISSA GOERKE ${ }^{5}$, SAMUEL DORSI ${ }^{5}$, JOSEPH UlaNOWSKI $^{6}$ \\ ${ }^{1}$ School of Earth and Environment and the National Centre for Atmospheric Science, University of Leeds, *Presenting Author \\ ${ }^{2}$ Cooperative Institute for Research in Environmental Science, University of Colorado \\ ${ }^{3}$ Aerospace Engineering Sciences, University of Colorado, ${ }^{4}$ NOAA/Earth System Research Laboratory, Boulder, Colorado \\ ${ }^{5}$ Polar Field Services, Littleton, Colorado \\ ${ }^{6}$ University of Hertfordshire
}

\begin{abstract}
A source of error in microphysical retrievals and model simulations is the assumption that clouds are composed of only randomly oriented ice crystals. This assumption is frequently not true, as evidenced by optical phenomena such as parhelia. Here, observations from the Cloud, Aerosol and Polarization Backscatter Lidar at Summit, Greenland are utilized along with other sensors and beam imaging to examine the properties of horizontally oriented ice crystals and the environment conditions in which they occur.
\end{abstract}

\section{INTRODUCTION}

The microphysical properties of clouds play a significant role in determining their radiative effect; one of these properties is the orientation of ice crystals. A source of error in current microphysical retrievals and model simulations is the assumption that clouds are composed of only randomly oriented ice crystals (ROIC). This assumption is frequently not true, as evidenced by optical phenomena such as parhelia (commonly referred to as sundogs). Here, observations from the Cloud, Aerosol and Polarization Backscatter Lidar (CAPABL) at Summit, Greenland are utilized along with instruments that are part of the Integrated Characterization of Energy, Clouds, Atmospheric state and Precipitation at Summit (ICECAPS) project in order to determine when, where and under what conditions horizontally oriented ice crystals (HOIC) occur at Summit, Greenland.

\section{METHOD}

Summit, Greenland is located $3.2 \mathrm{~km}$ above mean sea level on the Greenland Ice Sheet (GrIS) at $72.6^{\circ} \mathrm{N}, 38.5^{\circ} \mathrm{W}[1,2,3]$. In 2010 , the ICECAPS project established an intensive cloud observatory at the station [1]. ICECAPS includes a wide range of instrumentation with the ability to capture turbulent and thermodynamic properties of the atmosphere and clouds. Specific instrumentation included in the ICECAPS suite be used as part of this study includes the Cloud Aerosol Polarization and Backscatter Lidar (CAPABL), millimeterwave cloud radar (MMCR) and twice-daily radiosonde launches [1].

In this work we build upon the initial results of [1], [2] and [3] to examine the occurrence of HOIC over Summit, Greenland using CAPABL and supporting observations. HOIC exhibit both non-zero values of depolarisation and diattenuation when observed in a non-zenith/nonnadir orientation (non normal to the crystal face) while ROIC will only exhibit non-zero values of depolarisation. CAPABL was developed using the lidar polarisation theory of Hayman and Thayer (2012) [4]. To identify HOIC, CAPABL makes observations of diattenuation and depolarisation $[2,3]$. CAPABL accomplishes this by observing multiple planes of linear polarization, and photon counting and analog detection schemes, to selfevaluate, correct, and optimize signal combinations to improve cloud classification. For more information on how CAPABL implements the theory of Hayman and Thayer (2012) to make observations of HOIC please see $[2,3]$. Using the measures of diattenuation and depolarisation ratio, CAPABL is the only instrument with the ability to unambiguously identify HOIC on an operational basis [2]. In addition to the novel polarimetric observations we also show results from opportunistic observations of images projected onto the snow-covered surface near CAPABL during the Arctic night that originate from scattering in CAPABL's transmitted beam. This 
provides a unique validation of CAPABL's primary observations.

\subsection{HOIC Occurrence Categorisation}

The main study period considers a 335-day period starting on July $2^{\text {nd }}, 2015$ and ending on May $31^{\text {st }}$, 2016. Throughout this period CAPABL sampled at a temporal resolution of $5 \mathrm{~s}$ per polarisation and a spatial resolution of $7.5 \mathrm{~m}$. For an overall improved signal-to-noise ratio we have integrated this raw data to have a resolution of $30 \mathrm{~m}$ and $20 \mathrm{~s}$ (80 seconds for a complete 4 polarisation retrieval of diattenuation). Approximately 5 minutes of data per day was missed at midnight UTC to allow for system diagnostics [3]. Observations from lower than $0.2 \mathrm{~km}$ above ground level (AGL) were disregarded in order to minimize the influence of HOIC in fog/diamond dust and blowing snow as well a systemic effects due to the overlap of CAPABL's laser and telescope [3]. An additional day (December $6^{\text {th }}, 2016$ ) of HOIC observations will also be considered in the discussion as a unique phenomenon was captured using CAPABL's laser and digital photography that provides additional information about the crystal habits during HOIC events.

Having established the temporal and spatial domain of the study, criteria were set to identify the thermodynamic phase and orientation of the observed ice using the method outlined by [3]. Depolarisation values set for the identification of liquid and ice is consistent with previous studies of Arctic cloud by [5], [6] and [3]. Values of combined diattenuation were computed by multiplying CAPABL's two diattenaution observations together, which have opposite sensitivity to systematic effects, in order to remove false positives within the data as described in [3]. The threshold value of 0.005 used in the diattentuation criteria here is slightly more stringent (i.e. $\sqrt{0.005>.05}$ ) than the value used by [2].

\subsection{Identification of Atmospheric Conditions}

Values of temperature, relative humidity and horizontal wind speed were obtained from twicedaily radiosonde launches at Summit. Data for all variables from radiosondes were linearly interpolated to correspond to the range and time resolutions of measurements made by CAPABL. From this, observations were linearly interpolated with time for each day, creating a grid with the same spatial and temporal domain as the CAPABL observations but containing radiosonde observations.

\subsection{Analysis of Temporal Variability}

The frequencies of HOIC occurrence were calculated using all available days for each month of the study period. Frequencies of occurrence (F) of each atmospheric condition were calculated for each month using Equation 1 where $\mathrm{N}$ denotes the number of detections identified as a result of the masking process for HOIC, ice and liquid, and $\mathrm{x}$ is either HOIC, ice or liquid interchangeably.

$$
F_{x}=\frac{N_{x}}{N_{\text {HOIC }}+N_{\text {ROIC }}+N_{\text {Liq }}} \times 100
$$

The number of detections of each data type identified as a result of the masking process was summed in order to establish the total number of quality-controlled measurements per month.

All days containing HOIC were examined and the nature of the occurrence was classified as cirrus, stratiform or falling/precipitating. These classifications were based on CAPABL's observation plus co-incident observations of reflectivity, vertical fall speeds and spectral width provided by the MMCR [1,7]. The role of liquid was not considered in the analysis of cirrus cloud as they are composed solely of ice crystals. Liquid was also not considered in the analysis of the falling/precipitating classification because liquid droplets within Arctic clouds are too small to be precipitated [8]. Having classified the occurrences of HOIC, the number of occurrences per classification was totalled. A day containing more than one classification was counted in each of the classifications within the day.

\section{RESULTS OF POLARIMETRIC OBERVATIONS}

HOIC were positively identified in cirrus cloud, stratiform cloud or precipitating from cloud on 86 days of the 335-day study period. Figure 1 shows an example product of the masking process and applied to CAPABL's data as well as the ancillary observations from the MMCR on April 22, 2016 from 00:00 to 24:00 UTC.

HOIC were observed on 3 occasions during this period. These occurrences were identified from the masking procedure (results shown in the 
middle panel of Figure 1) and inspection of the corresponding radar observations in Figure 1. At $\sim 16: 00$ there is substantial HOIC present and this has been separated into 3 distinct occurrences using the combination of CAPABL and NOAA MMCR observations shown in Figure 1: 1) at $4 \mathrm{~km}$ a large pocket of HOIC is identified in cirris, 2) in small deck of liquid bearing stratiform cloud at $1.5 \mathrm{~km}$ and 3 ) in precipitating ice near the surface. Following these occurences HOIC was also observed at 18:00 in precipitating/falling ice that is connected to the higher clouds. Finally, at 20:00 and into the next day, the cirrus clouds at $4 \mathrm{~km}$ show a strong HOIC signal that is typical of HOIC in cirrus found on other days. This method is used throughout the study to broadly classify the type of HOIC occurrence.

Combining the HOIC mask with the information provided by interpolated radiosonde profiles bounding the observaiton period (for this example for profiles from $12 Z$ on April 22, 2016 and $00 \mathrm{Z}$ on April 23, 2016 were used) we create a pixel by pixel comparison of the conditions in which HOIC compared to ROIC occurred througout the day. For this example, we have not broken this into the classifications of cirrus, striatiform or precipitating ice due to the limited sample size. From the boxplots of Figure 2 it can be said the HOIC are occurring over a much smaller range of temperatures than ROIC, and a slightly higher temperature of $-25.4^{\circ} \mathrm{C}$ as opposed to the $-26.9^{\circ} \mathrm{C}$ of ROIC. The range of relative humidity (RH) over which HOIC are occurring is much wider than that of ROIC. HOIC also has a higher median value of $57.2 \%$ compared to that of the ROIC, 51.2\%. HOIC also have a lower median horizontal wind speed of $18.5 \mathrm{~ms}^{-1}$ as opposed to $19.6 \mathrm{~ms}^{-1}$ of ROIC. Further differences can be seen in the height ranges of both ROIC and HOIC. ROIC occurred over a larger vertical range than HOIC, and typically occurred higher in the atmosphere with a median height of $1.45 \mathrm{~km}$, compared to the $0.96 \mathrm{~km}$ of HOIC.

The overall results of the occurrence and classification analysis are summarized by month in Figure 3. Examining the entire data set in a manner similar to Figure 2, we find that the temperature of HOIC in all cases is significantly higher than that of ROIC. We also find that the mean RHs are higher for HOIC than they are for
ROIC in all cases. The mean horizontal wind speeds that HOIC occur under are always significantly higher than those of ROIC. Finally, the mean heights of HOIC in all cases were always significantly lower than that of ROIC.
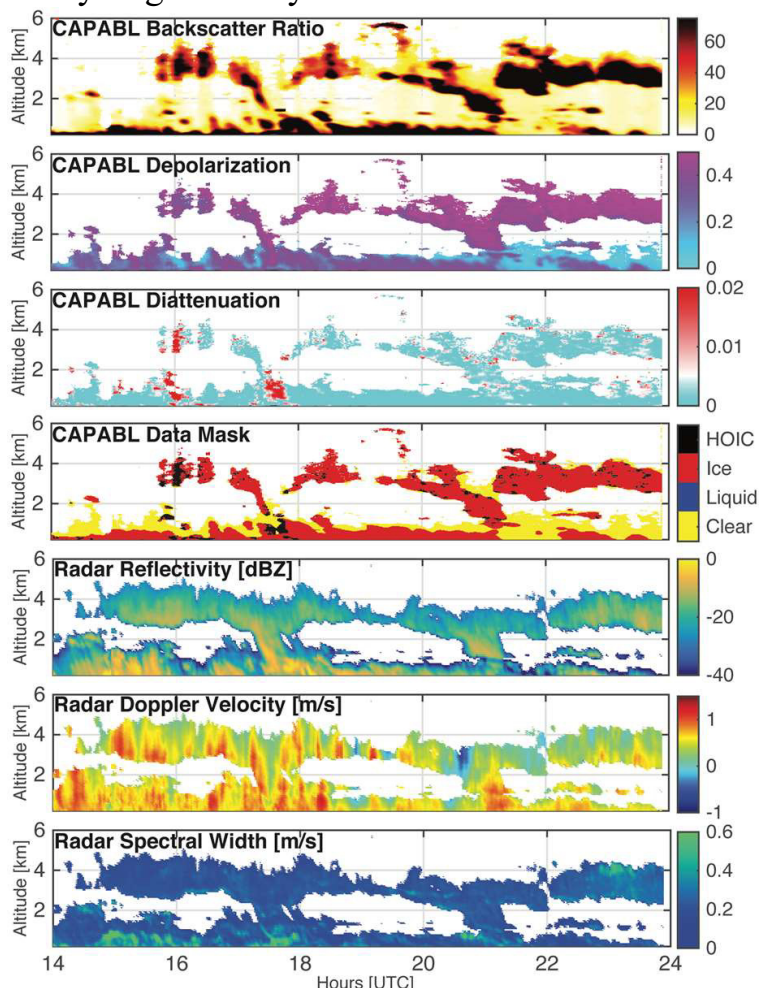

Figure 1: Observations made on from 14:00 to 24:00 UTC on April 22, 2016 from CAPABL and NOAA MMCR. Positive values in the Doppler velocity indicate a downward direction. The red colouring in the diattenuation plot exceed the 0.005 threshold for HOIC. HOIC occurs at 16:00 in cirrus at $4 \mathrm{~km}$, in stratiform cloud at $1.5 \mathrm{~km}$ and in precipitating ice near the surface, at 18:00 in precipitating/falling ice and at 20:00 onwards in cirrus cloud at $4 \mathrm{~km}$.
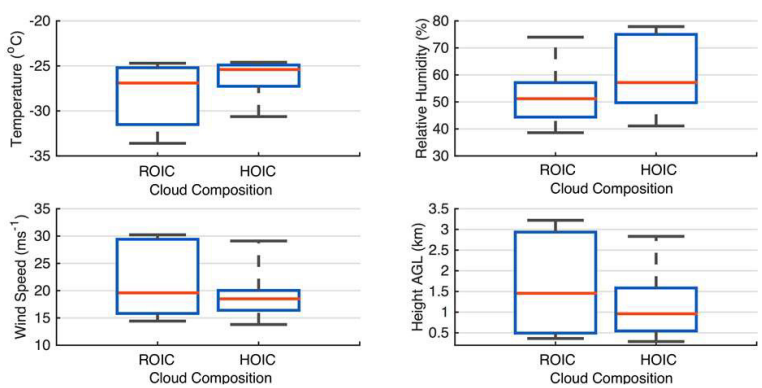

Figure 2: Comparison of condition in which ROIC and HOIC occurred in Figure 1 using CAPABL's masking from Figure 1 and interpolated radiosonde profiles launched $12 \mathrm{Z}$ on April 22, 2016 and 00Z on April 23, 2016. Boxes include the $25^{\text {th }}$ to $75^{\text {th }}$ percentiles and whiskers extend to the $10^{\text {th }}$ and $90^{\text {th }}$ percentiles. 


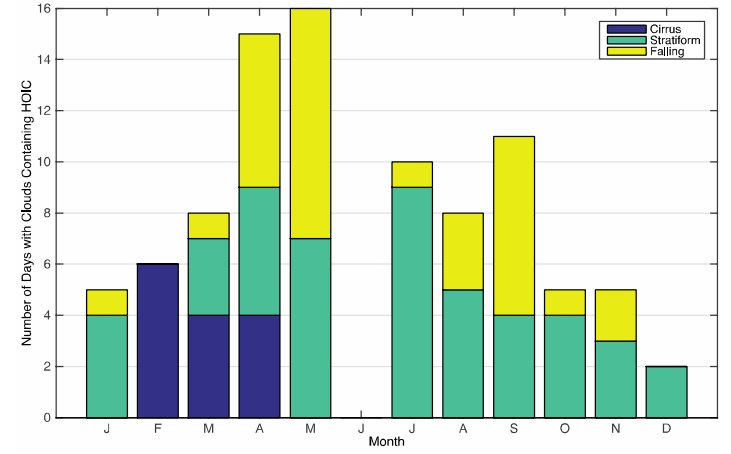

Figure 3: Monthly totals of days containing HOIC. Monthly totals are split into the 3 categories of cirrus, stratiform of falling/precipitated HOIC. June is not considered and therefore has no data.

\section{UNIQUE VALIDATION OF CAPABL'S HOIC CLASSIFICATION}

Ongoing work to understand HOIC at Summit includes recent observations of a phenomenon manifesting itself as succinct flashes of scattered light originating from CAPABL's transmit beam on the snow surface near the building housing the instrument (Figure 4). When photographed, the flashes reveal 2-D pattern similar to the forward scattering used to characterize cloud and aerosol particles. Using this information, theoretical 2-D patterns were computed using the ADDA discrete dipole approximation [9]. The simulations reveal that the flashes are originating from HOIC with large "flutter" angles $\left(5^{\circ}-8^{\circ}\right)$. The modelling also reveals that the population of ice crystals consists of $100-300 \mu \mathrm{m}$ pristine hexagonal plates, as well as much larger $(1-4 \mathrm{~mm})$ more irregular plates and columns. These observations qualitatively agree with CAPABL's and the MMCR's observations. More work will be done to make a quantitative comparison.

\section{DISCUSSION AND CONCLUSIONS}

The observations presented here provide the first documentation of the conditions under which HOIC occur at Summit, Greenland and are the first steps in the development of a climatology. This study has shed light on when and where HOIC are occurring but is subject to limitations such as poor resolution of the temperature and humidity observations used; these limitations however can be overcome with more and new observations. Future work will investigate the role of turbulence in the formation of HOIC by utilizing the observations from the MMCR. Sampling of temperature, water vapour and horizontal wind at a higher temporal resolution would also be beneficial for future research. Analysis of temporal variabilities in future work will extend and the study period used here and be used to explore the radiative properties of HOIC, especially their long wave effect.

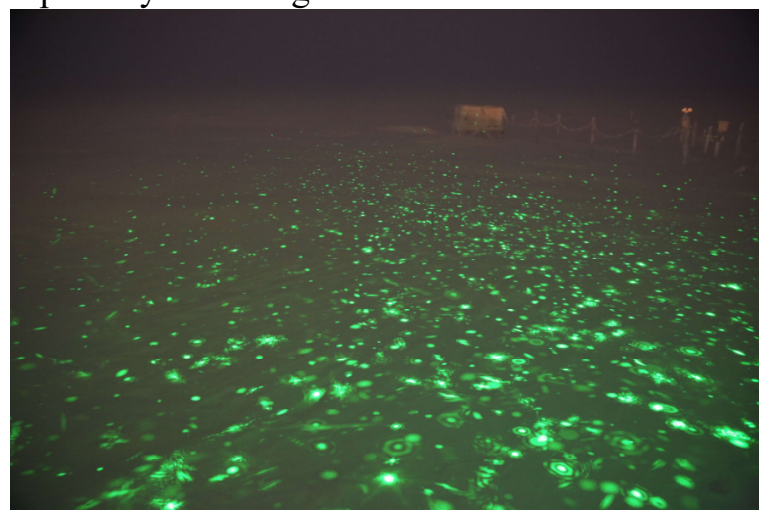

Figure 4: Scattering from the CAPABL's transmit beam onto snow from HOIC. This image consisits of 30 superimposed images each at $1.3 \mathrm{~s}$ exposure time. Recorded on 6 December 2016. CAPABL is behind the camera.

\section{ACKNOWLEDGEMENT}

This material is based upon work supported by the National Science Foundation Graduate Research Fellowship Program under Grant No. DGE 1144083, National Science Foundation Grants PLR-1303864, PLR-1303879, PLR-1314156, and ATM-0454999. All the authors would like to especially thank the staff and science technicians at Summit Station as well as the entire team at Polar Field Services for their support and dedication to help maintain the instrumentation and collect the data presented in this work.

\section{REFERENCES}

[1] Shupe, M. D., et al.: BAMS, 94, 169-186, 2013.

[2] Neely, R. R., et al.: JTECH, 1635-1655, 2013.

[3] Stillwell, R. A., et al.: Atmos. Meas. Tech. Discuss., in review, 2016.

[4] Hayman and Thayer: JOSAA, 400-409, 2012.

[5] Intrieri, J., et al.: J. Geo. Res., 2002.

[6] Shupe, M. D., and Uttal, T.: J. App. Met., 1544-1562, 2005.

[7] Moran, K.P., et al.: BAMS, 79, 443-455, 1998.

[8] Morrison, H., et al.: Nat. Geo., 5, 11-17, 2012.

[9] Yurkin, M. A. and Hoekstra, A. G.: J. Quantit.

Spectr. Rad. Transf. 112, 2234-2247 (2011). 